\title{
Temporal reward discounting in children, adolescents, and emerging adults during an experiential task
}

\author{
Anouk Scheres ${ }^{1,2 *}$, Chandra Tontsch ${ }^{1}$, Allison L. Thoeny $^{1}$ and Motofumi Sumiya ${ }^{1,3}$ \\ ${ }^{1}$ Department of Psychology, University of Arizona, Tucson, AZ, USA \\ 2 Behavioural Science Institute, Radboud University, Nijmegen, Netherlands \\ ${ }^{3}$ Division of Cerebral Integration, National Institute for Physiological Sciences, Okazaki, Japan
}

Edited by:

Frederic Dick, University of California, San Diego, USA

Reviewed by:

Frederic Dick, University of

California, San Diego, USA

Dima Amso, Brown University, USA

*Correspondence:

Anouk Scheres, Behavioural Science Institute, Radboud University,

Postbus 9104, 6500 HE, Nijmegen,

Netherlands

e-mail: a.scheres@psych.ru.nl
The goal of this study was to examine age effects on the ability/willingness to wait for large rewards in a real temporal reward discounting task from childhood to adulthood. Therefore, a real temporal discounting (TD) task was administered to children aged 6-12 $(n=39)$, adolescents aged 13-17 $(n=28)$, and young adults aged $18-19(n=55)$. Findings indicated that the cross-sectional development of TD followed a quadratic pattern across age groups, with adolescents choosing more often than children and adults to wait for the large delayed reward, resulting in reward-maximization. Various interpretations of this finding were offered, including a focus on reward maximization despite an immature ability to exert self-control, and flexible self-control which was high during this task as a result of strong motivation to maximize financial gains.

Keywords: adolescence, reward, temporal reward discounting, delay discounting, impulsivity, self-control

\section{INTRODUCTION}

Trading the anticipated benefits and costs of two choice options at different points in time is an important skill that we need to navigate through life: balancing the pursuit of future goals with enjoyment of the present moment, choosing between a high-fat dinner which you may enjoy and a perhaps less-preferred but healthier salad, deciding whether to save money for retirement or spending most of it now. When this balancing of short-term smaller gains and long-term larger gains is measured in laboratory settings, this is typically done by the use of temporal reward discounting tasks. Temporal discounting (TD) may be defined as the decrease of the subjective value of a (often monetary) reward as a function of pre-reward delay durations (Rachlin, 1989; Ainslie, 1992; Green and Myerson, 1993; Critchfield and Kollins, 2001). Put simply, the subjective value of a reward typically decreases the longer one has to wait for it. The rate at which this discounting takes place is measured by observing preferences when presented with choices between small immediate rewards and larger delayed rewards. In these choices, the large reward is usually kept constant (for example \$100), while the small reward varies (e.g., $\$ 10 / 20 / 30 / 40 / 50 / 60 / 70 / 80 / 90)$. The delays preceding the large reward are also varied (e.g., 1/30/90/180/365 days). For each delay, the large reward is paired with each of the small rewards and presented as a choice. For example, one choice may be "would you rather receive $\$ 50$ today or $\$ 100$ after 30 days?" By observing for each delay how large the immediate reward needs to be for a participant to be indifferent between the two options, one can then plot a discounting function indicating how rapidly the subjective value of a large reward decreases as a function of waiting time (see Critchfield and Kollins, 2001; Scheres et al., 2013). Generally, strong preferences for small immediate rewards result in steep TD functions (small area under the curve), while strong preferences for large delayed rewards result in shallow TD functions (large area under the curve).

The ability/willingness to forego smaller immediate rewards in favor of larger delayed rewards varies across individuals and is especially weak in those with psychiatric conditions with a childhood or adolescence onset, such as AttentionDeficit/Hyperactivity Disorder and Substance Abuse (SonugaBarke et al., 2008; de Wit, 2009; Luman et al., 2010). Additionally, the seminal work by Mischel et al. (2011 for a review), which utilizes the classic delay of gratification paradigm, has shown that the ability/willingness to wait at a young age predicts success in the academic, social, cognitive, and emotional domains during adolescence and adulthood. However, while the ability/willingness to wait has been shown to increase from young adulthood to old age (e.g., Green et al., 1994; Jimura et al., 2011; see also Green et al., 1996), our knowledge about how this develops from childhood into adulthood is still limited. This is because studies are relatively few (Scheres et al., 2006; Olson et al., 2007; Steinberg et al., 2009; Prencipe et al., 2011; Demurie, 2012; de Water et al., 2014), task formats vary across studies (hypothetical, potentially real, and real), and the majority of studies only included a relatively small age range (Scheres et al., 2006; Prencipe et al., 2011; Demurie, 2012; de Water et al., 2014), therefore not giving insight into the development from childhood into adulthood. One important reason to understand this developmental pathway in healthy populations is that it will provide a reference with which to compare individuals with childhood/adolescence-onset clinical conditions, such as ADHD and substance abuse.

Thus far, studies examining age effects on TD have primarily relied on tasks in which waiting times do not need to be endured and rewards are not actually paid to participants. Administering such a hypothetical task with large rewards of $\$ 1000$ 
and delays ranging from 1 day to 1 year to 935 adolescents and adults, Steinberg et al. (2009) reported that individuals aged 13 and younger discounted delayed rewards significantly more steeply than individuals aged 16 and older, with the 14-15 years olds falling in between. Similarly, Christakou et al. (2011) reported a linear increase from adolescence to adulthood in ability/willingness to wait for the large reward in a hypothetical TD paradigm with $\$ 100$ as the large reward and delays between 1 week and 1 year. Finally, Demurie (2012) used a hypothetical TD task with smaller money amounts and shorter waiting times: the large reward was 30 euro, and waiting times varied between 1 day and 2 weeks. They observed an increase in ability/willingness to wait for 30 euro from 8-10 to 11-13 years old, but the 14-16 years olds fell somewhere in between the 8-10 and 11-13 years olds. This suggests an inverted U-shaped relation between age and ability/willingness to wait, with maximal ability/willingness to wait in 11-13 years olds.

Four studies examining age effects on TD made use of potentially real tasks. In these tasks, participants are informed that one choice will be randomly selected and paid to the participant (Olson et al., 2007; Prencipe et al., 2011; de Water et al., 2014), or that there is a one in six chance that one choice will be randomly selected and paid to the participants (Audrain-McGovern et al., 2009). This design relies on the assumption that participants will choose on each trial pair as if it will have real consequences. In three of these studies, the large reward was $\$ 10$ and delays preceding this reward ranged from 1 day to 1 year. A linear increase in ability/willingness to wait from 8-9 years of age to $14-15$ years of age was observed (Prencipe et al., 2011), and from adolescence to adulthood (Olson et al., 2007; de Water et al., 2014). In the third study that used a potentially real task (Audrain-McGovern et al., 2009), the large reward varied between $\$ 25$ and 85 . The delays preceding this reward varied between 1 week and 6 months. Additionally, this study was unique in that it used a longitudinal (as opposed to cross-sectional) design, following participants from age 15 to 20, with data collected once every year. TD was found to be stable.

Finally, only in one study by Scheres et al. (2006), a real, experiential task was used, with large rewards of 10 dollar cents and actual waiting times between 5 and $30 \mathrm{~s}$. Delays were endured by participants and money amounts were really paid to participants. With this task, Scheres et al. reported that 6-11 years olds discounted delayed rewards more strongly than 12-17 years old, suggesting a linear increase in the ability/willingness to wait from childhood into adolescence.

Taking these studies together, we can conclude that there is a clear need for studies with more ecologically valid tasks in which a wider age range is included in order to enable researchers to examine whether decreases in discounting from childhood to young adolescence persist into older adolescence and emerging adulthood, or whether non-linear patterns may be observed from childhood into young adulthood. When summarizing the results from current studies, we can conclude that findings are mixed: age effects were reported to be linear, non-linear, and non-existent. Explanations for these inconsistencies may be found in the fact that most researchers may only have examined linear effects and not non-linear effects as well, in the different age ranges used, and in potentially meaningful differences in the specific TD task parameters such as magnitude of the rewards, duration of the delays, and whether the task was hypothetical, potentially real, or real. Most studies relied on hypothetical or potentially real tasks, limiting the ecological validity. The age range in most studies was restricted: only children and adolescents were included, or adolescents and adults, while only two studies enrolled children, adolescents, as well as adults (Olson et al., 2007; Steinberg et al., 2009). These latter two studies were limited in terms of ecological validity because they did not use real tasks. Previous work has shown that changes in task parameters and format have significant effects on the degree of discounting (Robles and Vargas, 2007, 2008; Tesch and Sanfey, 2008; Robles et al., 2009; Rodzon et al., 2011). Therefore, it is likely that differences in results are partly related to task differences.

More specifically, one may hypothesize that the findings depend on the relative contribution of self-control vs. affective processes to the choices made during the specific task. Although traditionally, the ability/willingness to wait for a large reward has been viewed as a sign of self-control, and a relative preference for small immediate rewards is often considered an index of impulsivity (e.g., Critchfield and Kollins, 2001; Green and Myerson, 2004), we suggest that TD may be better viewed as reflecting a trade-off between the ability to wait (self-control), and affective processes such as the sensitivity to the immediacy of the small reward and sensitivity to the magnitude of the delayed reward. Concretely, in order to wait for the larger delayed reward, an individual must be both willing and able to endure the waiting time, and be sufficiently motivated by/interested in the large reward as compared to the smaller, immediate alternative, to make the waiting worthwhile (see Marco et al., 2009; Kable, 2010; Scheres et al., 2010a for related discussions). Importantly, the relative contribution of self-control functions and affective processes in TD tasks may vary depending on the specific task parameters chosen. For example, in hypothetical tasks and/or in tasks with long delay durations, affective processes may play a minor role while cognitive control function may play a primary role. Therefore, the linear age effects which were mostly reported in studies with hypothetical tasks and long delay durations might be due to improvements in cognitive control functions during adolescence (Ridderinkhof and van der Molen, 1997; Williams et al., 1999; Luna et al., 2004; van den Wildenberg and van der Molen, 2004; Luciana et al., 2005; Huizinga et al., 2006; Bunge and Wright, 2007; Crone, 2009; Mischel et al., 2011). Whether studies with real tasks and/or short delay durations, which may rely more heavily on affective processes, will find linear or non-linear age effects in a sample spanning childhood to adulthood is an empirical question.

Therefore, the current study had three primary aims. First, we focused on a relatively wide age range, examining age effects on TD by including children, adolescents, as well as emerging adults. To this end, we included data from participants in these three age groups that were previously collected as part of larger studies (Scheres et al., 2006, 2008, 2010a,b). All participants were administered the same task. Secondly, we chose to use a real task in which participants experienced the consequences of their choices during the experiment: They endured the waiting times and received the rewards (money amounts displayed on the screen, and the actual sum of money paid at the end of the experiment). This is a clear 
advantage in terms of ecological validity, especially for the age range used here (see Navarick, 2004; Reynolds and Schiffbauer, 2004; Lagorio and Madden, 2005; Scheres et al., 2010b). While in real tasks, the reward magnitudes are necessarily small (typically up to $\$ 1$ ) and delay durations relatively short (up to $60 \mathrm{~s}$ ), these tasks have been proven to be useful in examining individual differences in children and adolescents. Additionally, the task is also a sensitive measure of discounting in young adults, as ceiling/floor effects have not been shown (Scheres et al., 2010b). The third aim of this study was to examine both linear and non-linear age effects on discounting.

Due to inconsistent findings in prior research, and based on different theoretical frameworks, several opposing hypotheses could be formulated. Discounting might be hypothesized to decrease linearly with age from childhood to adolescence and emerging adulthood, based on previous findings (Scheres et al., 2006; Olson et al., 2007; Steinberg et al., 2009), and based on the assumption that TD tasks primarily tap into cognitive control abilities which follow a protracted developmental pattern (Ridderinkhof and van der Molen, 1997; Williams et al., 1999; Luna et al., 2004; van den Wildenberg and van der Molen, 2004; Luciana et al., 2005; Huizinga et al., 2006; Bunge and Wright, 2007; Crone, 2009; Mischel et al., 2011). In contrast, non-linear age-related changes in TD, with a peak ability/willingness to wait during adolescence could be predicted based on one previous study (Demurie, 2012), and based on the notion that tasks with short delays and real rewards may trigger a relatively heavy involvement of affective processes, which play a unique role during adolescence (Ernst et al., 2005, 2006; Steinberg, 2005, 2008, 2010; Galvan et al., 2006; Casey et al., 2008a,b; Somerville et al., 2010; van Leijenhorst et al., 2010a,b; Crone and Dahl, 2012). A third possibility is that discounting remains stable, although previous work reporting on the lack of age effects was limited to 15-20 years olds (Audrain-McGovern et al., 2009). In order to address these competing hypotheses, we tested both linear and non-linear (i.e., quadratic) age effects in this study.

\section{MATERIALS AND METHODS PARTICIPANTS}

Three groups participated: children, adolescents, and young adults. The child group consisted of 39 typically developing 612 year olds ( 25 male, 14 female; mean age $9.3, S D=1.9$ ), and the adolescent group consisted of 28 typically developing 13-17 year olds (17 male, 11 female; mean age $14.4, S D=1.2)$. Both of these groups participated in previous research in which they were compared to children and adolescents with ADHD (Scheres et al., 2006, 2010a). The young adult group consisted of 55 undergraduate psychology students (26 male, 29 female; mean age 18.2, $S D=0.4)$ (see Scheres et al., 2008, 2010b).

\section{TASK}

Participants played a TD task in which they were instructed to make repeated choices between a small variable reward $(2,4,6$, or 8 cents) that would be delivered after $0 \mathrm{~s}$ and a large constant reward (10 cents) that would be delivered after a variable delay $[5,10,20,30$, (or 60) s]. For example, on some trials, participants had to choose between 6 cents now or 10 cents after waiting
$20 \mathrm{~s}$. Trials were administered in the same pseudo-random order to all participants. Each small immediate reward was paired twice with every delay for the large reward, resulting in a total of 40 choice trials. Choices were visually represented by two airplanes on a computer screen; each airplane carried their corresponding quantity of money, which was represented by a number and that specific amount of coins. Delays were represented by the level that the airplanes were flying - the higher the plane, the longer the delay duration (see Figure 1). The left or right position of the delayed reward plane was balanced over trials. Participants made a choice by pressing the button corresponding to the preferred plane (right or left) which resulted in the plane "dropping" its money into the participant's money basket on the bottom of the computer screen, either immediately or after the appropriate delay. Before the next trial the computer visually updated the total number of cents won. Participants were informed of the number of trials they would be presented with. Importantly, participants were not informed about the durations of the delays. Instead, they endured each delay during task practice, giving them a sense of the delay duration associated with each airplane level, without revealing the actual duration. After task completion, participants received the total amount of money won.

\section{PROCEDURE}

The study was approved by the Human Subjects Protection Program of the University of Arizona and New York University School of Medicine, and all participants provided prior written informed assent and consent.

\section{DATA PREPROCESSING}

Data were preprocessed based on previously reported procedures (Myerson et al., 2001; Scheres et al., 2006). Two independent raters determined subjective values for the delayed reward for each delay. Subjective value was defined as the magnitude of the small immediate reward for which the participant showed indifference in a choice against the large delayed reward (see Critchfield and Kollins, 2001; Scheres et al., 2006). Between-rater agreement was very good (mean kappa 0.90 , range $0.72-1.00$ ). In rare cases of disagreement, a consensus on subjective value was reached by discussion. The second step was to calculate area under

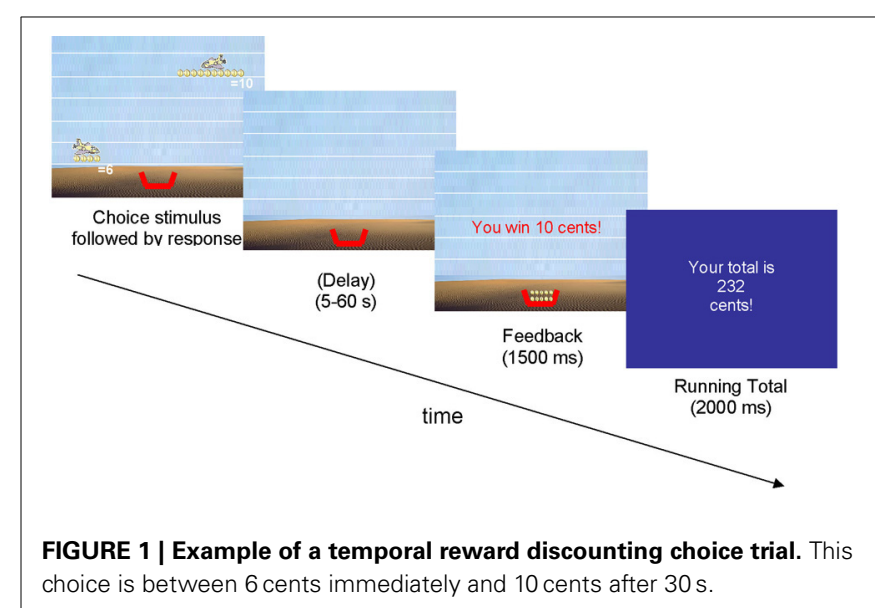


the discounting curve (AUC) for the TD functions (following the procedure described by Myerson et al., 2001, and used by Scheres et al., 2006, 2010a,b). First, subjective values and delays were normalized. That is, subjective values were expressed as proportions of the amount of the maximum delayed reward, and delays were expressed as proportions of the maximum delay. In this case, since the delays ranged from 5-30 in one dataset (Scheres et al., 2006) and from 5-60 in the other datasets (Scheres et al., 2010a,b), $30 \mathrm{~s}$ was used as the maximum delay for the purpose of delay standardization (see Myerson et al., 2001). The normalized values were used as $x$ and $y$ coordinates ( $x=$ delay; $y=$ subjective value). The data points on the $y$ axis were connected, thus forming the discounting function. From each standardized subjective value, vertical lines were drawn to determine separate trapezoids. The area of each trapezoid equals $(x 2-x 1) *[(y 1+y 2) / 2]$, where $x 1$ and $x 2$ are successive delays, and $y 1$ and $y 2$ are the subjective values associated with these delays. Using this formula, the area of each trapezoid was calculated and subsequently the areas were summed, resulting in the dependent variable of interest: total AUC which has a range of $0-1$. In general, a smaller AUC reflects a steeper discounting function (i.e., less willingness to wait as the delay duration increases).

\section{STATISTICAL ANALYSES}

\section{Age group comparisons}

ANOVAs were conducted with AUC as the dependent variable and age group with three levels (children, adolescents, emerging adults) as the between subject factor. Significant main effects of group in the ANOVA were followed by contrast analyses to examine the shape of the age effect.

\section{Regression analyses}

Because the group stratification by age may be viewed as arbitrary, we also used linear regression to examine the continuous relation between age and AUC. In order to examine linear effects, age was entered as the predictor and AUC as the dependent variable. In order to test for non-linear (quadratic) age effects, age $\times$ age (after centering age around its mean) was entered as a predictor, with AUC as the dependent variable. Finally, in order to test whether there was a meaningful difference in the explanatory power of linear vs. quadratic effects, age was entered as a predictor at step 1 , followed by age $\times$ age at step 2 .

\section{RESULTS}

ANOVA showed that there was a significant, medium-sized effect of age group on AUC when comparing children, adolescents, and adults $\left[F_{(2,121)}=7.4, p<0.001\right.$; partial $\left.\eta^{2}=0.11\right]$. Subsequent contrast analysis revealed that the shape of this age effect was best described as quadratic ( $p<0.001$, as compared to $p<0.08$ for linear), with an increase in AUC from childhood $(M=0.49$, $S E=0.03)$ to adolescence $(M=0.67, S E=0.04)$, followed by a decrease in AUC from adolescence to emerging adulthood $(M=$ $0.56, S E=0.03)$. Figure 2 displays the discounting curves for each age group.

Regression analysis showed that there was a small-sized linear increase of AUC with increasing age that fell short of statistical significance $\left(r=0.17 ; p<0.058, R^{2}=0.03\right)$. The quadratic relation between age and AUC, however, was significant and medium-sized $\left(r=-0.32 ; p<0.001, R^{2}=0.10\right)$. The ability/willingness to wait appeared to peak around age 14 (see Figure 3). The quadratic effect added explanatory power over and beyond the linear effect $\left(\Delta R^{2}=0.08 ; p<0.002\right)$.

\section{DISCUSSION}

This study had as a goal to examine whether age effects on temporal reward discounting (TD) with a real task with relatively small money amounts and short waiting times were linear or quadratic in a group of participants spanning childhood to emerging adulthood (6-19). To this end, we analyzed data from a real TD task that was administered to children, adolescents, and young adults (Scheres et al., 2006, 2008, 2010a,b). We tested both linear and quadratic effects of age on AUC. The findings did not support the hypothesis of a linear relation between age and TD, but did

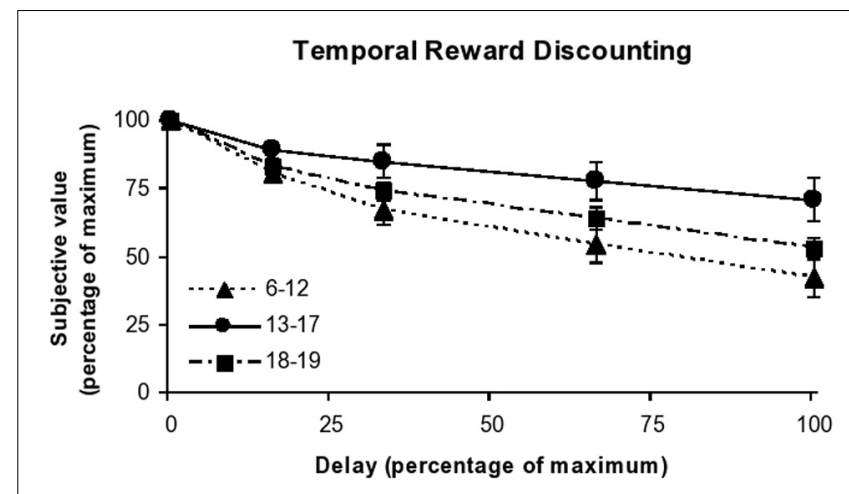

FIGURE 2 | Temporal reward discounting functions for children, adolescents, and young adults.

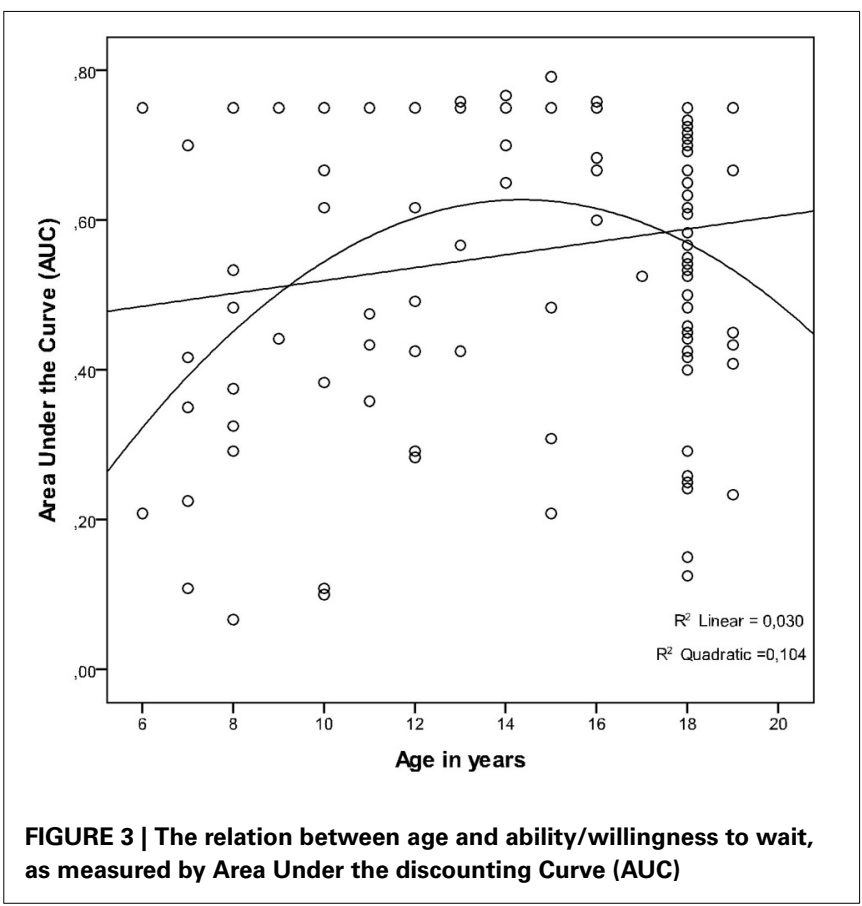


support the hypothesis that of a quadratic relation between age and TD, with the ability/willingness to wait for large rewards peaking during adolescence, in particular at the age of 14 .

At first, this may seem a counter-intuitive finding because adolescents are often thought of as impulsive, while choosing to maximize rewards relatively often as they did on this task is typically viewed as the opposite of impulsive, namely as a sign of self-control (e.g., Critchfield and Kollins, 2001; Green and Myerson, 2004; Mischel et al., 2011). For instance, Mischel et al. (2011) have proposed that the ability to resist temptation in favor of long-term goals, also referred to as "willpower," self-control, or a variety of inhibitory processes, is the key ingredient which determines to what extent a participant is able to overcome a tempting immediate reward (see also Figner et al., 2010). However, we note here that inhibitory control has been shown to develop linearly from childhood into adulthood (Ridderinkhof and van der Molen, 1997; Williams et al., 1999; Crone, 2009). On the contrary, our data show a non-linear developmental pattern for TD. Thus, we suggest here that multiple processes are important determinants of choice preference in TD tasks, with inhibition being only one of them. The following factors also play an important role: sensitivity to reward immediacy, sensitivity to reward magnitude, and delay aversion (see Marco et al., 2009; Scheres et al., 2010a).

Therefore, based on the idea that it is useful to think of selfcontrol and various reward aspects as playing complementary and interactive roles in TD paradigms, we propose the following interpretation of the data: Choices made on TD paradigms likely result from a balance between affective processes including delay aversion and sensitivity to various reward aspects (such as immediacy of the reward, and magnitude of the reward) on the one hand, and self-control on the other. This balance may be biased toward affective, reward-related aspects or toward selfcontrol, depending on factors such as age and context (e.g., is the task real or hypothetical). Our data show that the balance gravitated toward "self-control" in adolescence, as compared to childhood and adulthood. However, this does not necessarily mean that self-control peaks in adolescence: On the contrary, there is ample evidence to suggest that self-control keeps improving well into adulthood (Williams et al., 1999; Crone, 2009). Rather, the current findings may suggest that a relatively high sensitivity to reward magnitude/maximization motivated adolescents to wait, despite their generally immature ability to exert selfcontrol. This interpretation is consistent with previous research which has demonstrated that reward-seeking or reward sensitivity peaks during early to middle adolescence (Galvan et al., 2006; Steinberg, 2008, 2010; Casey et al., 2008a,b), and that reward- and emotion- related brain regions (for example, the nucleus accumbens) are relatively mature during adolescence (e.g., Casey et al., 2008a,b; Steinberg, 2008; see also Ernst et al., 2005, 2006; Galvan et al., 2006; Galvan, 2010; Steinberg, 2010; van Leijenhorst et al., 2010a,b). More generally, it is consistent with the idea that early to middle adolescence is a unique developmental period.

Alternatively, it may be suggested that rather than the ability to exert self-control being statically immature during adolescence, this ability may flexibly fluctuate, depending on contextual, social, and emotional factors. In this case, it may be hypothesized that adolescents exerted great self-control because they were highly motivated to maximize their financial gains (see Crone and Dahl, 2012 for a recent theoretical framework). In a different context, such as in a hypothetical task in which the money would not be paid to the participants, the motivation to exert self-control may be lower, and a different developmental pattern for TD may be found. This may be a plausible explanation for the fact that most studies with hypothetical or potentially real TD tasks have reported linear effects of age on the ability/willingness to wait (Olson et al., 2007; Steinberg et al., 2009; Christakou et al., 2011; Prencipe et al., 2011; de Water et al., 2014).

More generally, we cannot assume that hypothetical and real TD tasks measure similar processes. Specifically, Navarick (2004) has suggested that hypothetical TD tasks may measure an entirely different discounting process than tasks using real rewards and real delays. However, note that experimental research has later demonstrated that previously found differences in discount rate between real and hypothetical tasks are attributable to differences in reward magnitudes and delay durations (Lagorio and Madden, 2005; Scheres et al., 2010b). It will be of interest to directly compare the developmental pattern of real and hypothetical discounting tasks with varying reward magnitudes and delay durations in future research. Additionally, adding tasks that measure self-control functions such as response inhibition and working memory, as well as tasks that tap into affective aspects such as reward valuation, will provide further insights.

A number of limitations need to be considered here. The possible interpretations of the quadratic age effect are still speculative, and future research is clearly needed to replicate and clarify these findings, including functional brain imaging work to determine whether these age effects can be in part explained by neuromaturational effects. Secondly, this study was aimed at exploring the developmental pattern of TD from childhood into young adulthood but did not include additional measures. We recommend that future research includes a behavioral measure of money valuation, to test whether the decrease in discount rate from adolescence to adulthood can be explained by differences in money valuation. Additionally, future research may implement task manipulations to specifically examine the relative contribution of the various reward aspects in this task such as sensitivity to reward immediacy, reward magnitude, and delay aversion (see Scheres et al., 2010b). More specifically, such research could include multiple versions of a TD task, such as with and without post-reward delays (see Sonuga-Barke et al., 1992; Scheres et al., 2006; Marco et al., 2009), and with varying reward magnitudes and number of trials (Scheres et al., 2010a) in order to achieve this goal. Finally, our goal is to follow this study by a larger lifespan development study on TD, which will provide us with a reference with which to compare the TD development of clinical populations, such as ADHD (see Paus et al., 2008).

In conclusion, this study showed that the cross-sectional development of TD from childhood into young adulthood as measured with a real task, follows a quadratic pattern. More specifically, the ability/willingness to wait peaked at age 14. Various interpretations of this finding were offered, including a focus on reward maximization despite an immature ability to exert self-control, and a high level of self-control which was motivated by the prospect of a maximum money amount. These data may provide 
a reference with which to compare the development of TD in clinical populations. Additional task manipulations as well as the use of functional brain imaging will help to further clarify the nature and biological basis of this finding.

\section{ACKNOWLEDGMENTS}

The authors are grateful to Bill Burk for his advice on statistical analyses.

\section{REFERENCES}

Ainslie, G. (1992). Picoeconomics. Cambridge: Cambridge University Press.

Audrain-McGovern, J., Rodriguez, D., Epstein, L. H., Cuevas, J., Rodgers, K., and Wileyto, E. P. (2009). Does delay discounting play an etiological role in smoking or is it a consequence of smoking? Drug Alcohol Depend. 103, 99-106. doi: 10.1016/j.drugalcdep.2008.12.019

Bunge, S. A., and Wright, S. B. (2007). Neurodevelopmental changes in working memory and cognitive control. Curr. Opin. Neurobiol. 17, 243-250. doi: 10.1016/j.conb.2007.02.005

Casey, B. J., Getz, S., and Galvan, A. (2008b). The adolescent brain. Dev. Rev. 28, 62-77. doi: 10.1016/j.dr.2007.08.003

Casey, B. J., Jones, R. M., and Hare, T. A. (2008a). The adolescent brain. Ann. N.Y. Acad. Sci. 1124, 111-126. doi: 10.1196/annals. 1440.010

Christakou, A., Brammer, M., and Rubia, K. (2011). Maturation of limbic corticostriatal activation and connectivity associated with developmental changes in temporal discounting. Neuroimage 54, 1344-1354. doi: 10.1016/j.neuroimage.2010.08.067

Critchfield, T. S., and Kollins, S. H. (2001). Temporal discounting: basic research and the analysis of socially important behavior. J. Appl. Behav. Anal. 34, 101-122. doi: 10.1901/jaba.2001.34-101

Crone, E. A. (2009). Executive functions in adolescence: inferences from brain and behavior. Dev. Sci. Rev. 12, 825-830. doi: 10.1111/j.1467-7687.2009.00918.x

Crone, E. A., and Dahl, R. E. (2012). Understanding adolescence as a period of social-affective engagement and goal flexibility. Nat. Rev. Neurosci. 13, 636-650. doi: $10.1038 / \mathrm{nrn} 3313$

Demurie, E. (2012). Sensitivity for Reward in Children with AttentionDeficit/Hyperactivity Disorder. Ghent: Ghent University, Department of Experimental Clinical and Health Psychology.

de Water, E., Cillessen, A. H., and Scheres, A. (2014). Distinct age-related differences in temporal discounting and risk taking in adolescents and young adults. Child Dev. doi: 10.1111/cdev.12245. [Epub ahead of print].

de Wit, H. (2009). Impulsivity as a determinant and consequence of drug use: a review of underlying processes. Addict. Biol. 14, 22-31. doi: 10.1111/j.13691600.2008.00129.x

Ernst, M., Nelson, E. E., Jazbec, S., McClure, E. B., Monk, C. S., Leibenluft, E., et al. (2005). Amygdala and nucleus accumbens in responses to receipt and omission of gains in adults and adolescents. Neuroimage 25, 1279-1291. doi: 10.1016/j.neuroimage.2004.12.038

Ernst, M., Pine, D. S., and Hardin, M. (2006). Triadic model of the neurobiology of motivated behavior in adolescence. Psychol. Med. 36, 299-312. doi: $10.1017 /$ S0033291705005891

Figner, B., Knoch, D., Johnson, E. J., Krosch, A. R., Lisanby, S. H., Fehr, E., et al. (2010). Lateral prefrontal cortex and self-control in intertemporal choice. Nat. Neurosci. 13, 538-539. doi: 10.1038/nn.2516

Galvan, A. (2010). Adolescent development of the reward system. Front. Hum. Neurosci. 4:6. doi: 10.3389/neuro.09.006.2010

Galvan, A., Hare, T. A., Parra, C. E., Penn, J., Voss, H., Glover, G., et al. (2006). Earlier development of the accumbens relative to orbitofrontal cortex might underlie risk-taking behavior in adolescents. J. Neurosci. 26, 6885-6892. doi: 10.1523/JNEUROSCI.1062-06.2006

Green, L., Fry, A., and Myerson, J. (1994). Discounting of delayed rewards: a life-span comparison. Psychol. Sci. 5, 33-36. doi: 10.1111/j.1467-9280.1994. tb00610.x

Green, L., and Myerson, J. (1993). Alternative frameworks for the analysis of selfcontrol. Behav. Philos. 21, 37-47.

Green, L., and Myerson, J. (2004). A discounting framework for choice with delayed and probabilistic rewards. Psychol. Bull. 130, 769-792. doi: 10.1037/00332909.130.5.769
Green, L., Myerson, J., Lichtman, D., Rosen, S., and Fry, A. (1996). Temporal discounting in choice between delayed rewards: the role of age and income. Psychol. Aging 11, 79-84. doi: 10.1037/0882-7974.11.1.79

Huizinga, M., Dolan, C. V., and van der Molen, M. W. (2006). Age-related change in executive function: developmental trends and a latent variable analysis. Neuropsychologia 44, 2017-2036. doi: 10.1016/j.neuropsychologia.2006.01.010

Jimura, K., Myerson, J., Hilgard, J., Keighley, J., Braver, T. S., and Green, L. (2011). Domain independence and stability in young and older adults' discounting of delayed rewards. Behav. Processes 87, 253-259. doi: 10.1016/j.beproc.2011. 04.006

Kable, J. W. (2010). Just a little (lateral prefrontal) patience. Nat. Neurosci. 13, 523-524. doi: 10.1038/nn0510-523

Lagorio, C. H., and Madden, G. J. (2005). Delay discounting of real and hypothetical rewards III: steady-state assessments, forced-choice trials, and all real rewards. Behav. Processes 69, 173-187. doi: 10.1016/j.beproc.2005.02.003

Luciana, M., Conklin, H. M., Hooper, C. J., and Yarger, R. S. (2005). The development of nonverbal working memory and executive control processes in adolescents. Child Dev. 76, 697-712. doi: 10.1111/j.1467-8624.2005.00872.x

Luman, M., Tripp, G., and Scheres, A. (2010). Identifying the neurobiology of altered reinforcement sensitivity in ADHD: a review and research agenda. Neurosci. Biobehav. Rev. 34, 744-754. doi: 10.1016/j.neubiorev.2009.11.021

Luna, B., Garver, K. E., Urban, T. A., Lazar, N. A., and Sweeney, J. A. (2004). Maturation of cognitive processes from late childhood to adulthood. Child Dev. 75, 1357-1372. doi: 10.1111/j.1467-8624.2004.00745.x

Marco, R., Miranda, A., Schlotz, W., Melia, A., Mulligan, A., Müller, U., et al. (2009). Delay and reward choice in ADHD: an experimental test of the role of delay aversion. Neuropsychology 23, 367-380. doi: 10.1037/a0014914

Mischel, W., Ayduk, O., Berman, M. G., Casey, B. J., Gotlib, I. H., Jonides, J., et al. (2011). "Willpower" over the life span: decomposing self-regulation. Soc. Cogn. Affect. Neurosci. 6, 252-256. doi: 10.1093/scan/nsq081

Myerson, J., Green, L., and Warusawitharana, M. (2001). Area under the curve as a measure of discounting. J. Exp. Anal. Behav. 76, 235-243. doi: 10.1901/jeab.2001.76-235

Navarick, D. J. (2004). Discounting of delayed reinforcers: measurement by questionnaires versus operant choice procedures. Psychol. Rec. 54, 85-94.

Olson, E. A., Hooper, C. J., Collins, P., and Luciana, M. (2007). Adolescents' performance on delay and probability discounting tasks: contributions of age, intelligence, executive functioning, and self-reported externalizing behavior. Per. Individ. Dif. 43, 1886-1897. doi: 10.1016/j.paid.2007.06.016

Paus, T., Keshavan, M., and Giedd, J. N. (2008). Why do many psychiatric disorders emerge during adolescence? Nat. Rev. Neurosci. 9, 947-957. doi: $10.1038 / \mathrm{nrn} 2513$

Prencipe, A., Kesek, A., Cohen, J., Lamm, C., Lewis, M. D., and Zelazo, P. D. (2011). Development of hot and cool executive function during the transition to adolescence. J. Exp. Child Psychol. 108, 621-637. doi: 10.1016/j.jecp.2010.09.008

Rachlin, H. (1989). Judgment, Decision and Choice. New York, NY: W. H. Freeman.

Reynolds, B., and Schiffbauer, R. (2004). Measuring state changes in human delay discounting: an experiential discounting task. Behav. Processes 67, 343-356. doi: 10.1016/j.beproc.2004.06.003

Ridderinkhof, K. R., and van der Molen, M. W. (1997). Mental resources, processing speed, and inhibitory control: a developmental perspective. Biol. Psychol. 45, 241-261. doi: 10.1016/S0301-0511(96)05230-1

Robles, E., and Vargas, P. A. (2007). Functional parameters of delay discounting assessment tasks: order of presentation. Behav. Processes 75, 237-241. doi: 10.1016/j.beproc.2007.02.014

Robles, E., and Vargas, P. A. (2008). Parameters of delay discounting assessment: number of trials, effort, and sequential effects. Behav. Processes 78, 285-290. doi: 10.1016/j.beproc.2007.10.012

Robles, E., Vargas, P. A., and Bejarano, R. (2009). Within-subject differences in degree of delay discounting as a function of order of presentation of hypothetical cash rewards. Behav. Processes 81, 260-263. doi: 10.1016/j.beproc.2009. 02.018

Rodzon, K., Berry, M. S., and Odum, A. L. (2011). Within-subject comparison of degree of delay discounting using titrating and fixed sequence procedures. Behav. Processes 86, 164-167. doi: 10.1016/j.beproc.2010.09.007

Scheres, A., Dijkstra, M., Ainslie, E., Balkan, J., Reynolds, B., Sonuga-Barke, E., et al. (2006). Temporal and probabilistic discounting of rewards in children and adolescents: effects of age and ADHD symptoms. Neuropsychologia 44, 2092-2103. doi: 10.1016/j.neuropsychologia.2005.10.012 
Scheres, A., de Water, E., and Mies, G. W. (2013). The neural correlates of temporal reward discounting. WIREs Cogn. Sci. 4, 523-545. doi: 10.1002/ wcs. 1246

Scheres, A., Lee, A., and Sumiya, M. (2008). Temporal reward discounting and ADHD: task and symptom specific effects. J. Neural Transm. 115, 221-226. doi: 10.1007/s00702-007-0813-6

Scheres, A., Sumiya, M., and Lee, A. (2010b). Studying the relation between temporal reward discounting tasks used in populations with ADHD: a factor analysis. Int. J. Methods Psychiatr. Res. 19, 167-176. doi: 10.1002/mpr.309

Scheres, A., Tontsch, C., Lee, A., and Kaczkurkin, A. (2010a). Temporal reward discounting in children and adolescents with ADHD: effects of symptom domains, reward magnitude, and session length. Biol. Psychiatry 67, 641-648. doi: 10.1016/j.biopsych.2009.10.033

Somerville, L. H., Jones, R. M., and Casey, B. J. (2010). A time of change: behavioral and neural correlates of adolescent sensitivity to appetitive and aversive environmental cues. Brain Cogn. 72, 124-133. doi: 10.1016/j.bandc.2009. 07.003

Sonuga-Barke, E. J. S., Sergeant, J. A., Nigg, J., and Willcutt, E. (2008). Executive dysfunction and delay aversion in attention deficit hyperactivity disorder: nosologic and diagnostic implications. Child Adolesc. Psychiatr. Clin. N. Am. 17, 367-384. doi: 10.1016/j.chc.2007.11.008

Sonuga-Barke, E. J. S., Taylor, E., Sembi, S., and Smith, J. (1992). Hyperactivity and delay aversion-I. the effect of delay on choice. J. Child Psychol. Psychiatry 33, 387-398.

Steinberg, L. (2005). Cognitive and affective development in adolescence. Trends Cogn. Sci. 9, 69-74. doi: 10.1016/j.tics.2004.12.005

Steinberg, L. (2008). A social neuroscience perspective on adolescent risk-taking. Dev. Rev. 28, 78-106. doi: 10.1016/j.dr.2007.08.002

Steinberg, L. (2010). A dual systems model of adolescent risk-taking. Dev. Psychobiol. 52, 216-224. doi: 10.1002/dev.20445

Steinberg, L., Graham, S., O’Brien, L., Woolard, J., Cauffman, E., and Banich, M. (2009). Age differences in future orientation and delay discounting. Child Dev. 80, 28-44. doi: 10.1111/j.1467-8624.2008.01244.x
Tesch, A. D., and Sanfey, A. G. (2008). Models and methods in delay discounting. Ann. N. Y. Acad. Sci. 1128, 90-94. doi: 10.1196/annals.1399.010

van den Wildenberg, W. P. M., and van der Molen, M. W. (2004). Developmental trends in simple and selective inhibition of compatible and incompatible responses. J. Exp. Child Psychol. 87, 201-220. doi: 10.1016/j.jecp.2003.11.003

van Leijenhorst, L., Moor, B. G., Op de Macks, Z. A., Rombouts, S. A. R. B., Westenberg, P. M., and Crone, E. A. (2010b). Adolescent risky decision-making: neurocognitive development of reward and control regions. Neuroimage 51, 345-355. doi: 10.1016/j.neuroimage.2010.02.038

van Leijenhorst, L., Zanolie, K., Van Meel, C. S., Westenberg, P. M., Rombouts, S. A., and Crone, E. A. (2010a). What motivates the adolescent? Brain regions mediating reward sensitivity across adolescence. Cereb. Cortex 20, 61-69. doi: 10.1093/cercor/bhp078

Williams, B. R., Ponesse, J. S., Schachar, R. J., Logan, G. D., and Tannock, R. (1999). Development of inhibitory control across the life span. Dev. Psychol. 35, 205-213.

Conflict of Interest Statement: The authors declare that the research was conducted in the absence of any commercial or financial relationships that could be construed as a potential conflict of interest.

Received: 12 February 2014; accepted: 19 June 2014; published online: 08 July 2014. Citation: Scheres A, Tontsch C, Thoeny AL and Sumiya M (2014) Temporal reward discounting in children, adolescents, and emerging adults during an experiential task. Front. Psychol. 5:711. doi: 10.3389/fpsyg.2014.00711

This article was submitted to Developmental Psychology, a section of the journal Frontiers in Psychology.

Copyright (C) 2014 Scheres, Tontsch, Thoeny and Sumiya. This is an open-access article distributed under the terms of the Creative Commons Attribution License (CC BY). The use, distribution or reproduction in other forums is permitted, provided the original author(s) or licensor are credited and that the original publication in this journal is cited, in accordance with accepted academic practice. No use, distribution or reproduction is permitted which does not comply with these terms. 